\title{
MicroRNA-151 regulates the growth, drug sensitivity and epithelial mesenchymal transition of human glioma cells by targeting profilin 2
}

\author{
Wenxue Zhang1', Hanhong Niu'1, Rongze Wang ${ }^{1}$, Chenchao Liu2,3 and Yi Wang ${ }^{2,3 凶}$ \\ 1Department of Radiation Oncology, Tianjin Medical University General Hospital, Tianjin, 300052, China; 2Department of Neurosurgery, Tianjin \\ Medical University General Hospital, Tianjin, 300052, China; ${ }^{3 T i a n j i n ~ N e u r o l o g i c a l ~ I n s t i t u t e, ~ T i a n j i n, ~ 300052, ~ C h i n a ~}$
}

\begin{abstract}
The microRNA-151 (miR-151) has been reported to be involved in the growth, development, and tumorigenesis of different types of human cancers. This study was designed to unravel the role and therapeutic potential of miR-151 in glioma. The results showed glioma was found to be associated with significant $(P<0.05)$ downregulation of miR-151. Low expression of miR-151 was also associated with poor survival of the glioma patients. Overexpression of miR-151 resulted in a significant $(P<0.05)$ decline of glioma cell proliferation and colony formation. The sensitivity of the glioma cells to adriamycin also increased significantly $(P<0.05)$ upon miR-151 overexpression. Additionally, overexpression of miR-151 also suppressed the migration and invasion of the human glioma cells. This was also associated with alteration in the expression of epithelial mesenchymal transition proteins. The expression of E-cadherin was increased while as that of $\mathrm{N}$-cadherin, vimentin, and Snail was considerably decreased upon miR-151 overexpression. Bioinformatic analysis and ducal luciferase assay showed miR-151 targets profilin 2 (PFN2) in human glioma cells. The expression of PFN2 was found to be significantly $(P<0.05)$ upregulated in human glioma tissues cells and cell lines. Nonetheless, the PFN2 expression was considerably suppressed upon miR-151 overexpression. Knockdown of PFN2 resulted in decrease of glioma cells proliferation. In contrary, overexpression of PFN2 could avoid the tumorsuppressive effects of miR-151. Taken together, present study points towards the tumor-suppressive effects of miR-151 and prospective therapeutic implications in human glioma.
\end{abstract}

Key words: Glioma, micro-RNA, adriamycin, epithelial-to-mesenchymal transition, migration, invasion.

Received: 09 July, 2021; revised: 24 July, 2021; accepted: 28 July, 2021; available on-line: 02 March, 2022

凶e-mail: ewang_2021@yeah.net

Acknowledgements of Financial Support: The authors acknowledge the funding support from the Foundation of Chinese Society of Neuro-Oncology, (Grant/Award Number: CSNO2013-MSD003), Wu Jieping Medical Fundation (Grant/ Award Number:320.6750.17137) and Key Science \& Technology Program of Tianjin Education Commission (Grant/Award number: 2016YD02). Abbreviations: MTT, 3-(4,5-dimethylthiazol-2-yl)-2,5-diphenyl tetrazolium bromide; EMT, epithelial-to-mesenchymal transition; PBS, phosphate buffered saline, PFN, Profilin 2

\section{INTRODUCTION}

The glioma, representing the malignant form of brain tumor originating from neuro-epithelial tissues, is con- sidered as the most dominant tumor of human brain (Schwartzbaum et al., 2006). Nearly 30\% cases of the total malignant tumors of central nervous system and more than $80 \%$ of intra-cranial tumor malignancies system are believed to correspond to glioma (Goodenberger \& Jenkins, 2012). Moreover, sufficient level of annual fatality is seen to be caused by human glioma (Shi et al., 2018). Although, the advanced surgical approaches along with chemo/radio-therapeutic modalities have reduced the proportion of annual glioma deaths, the mortality data still demand exploration of better treatment strategies. There are scientific reports that highlight the role of micro-RNAs (miRs) as effective prognostic and therapeutic agents against cancer (To et al., 2018). The miRs which represent a heterogenous class of endogenously synthesized RNA oligonucleotides act at the translational level to regulate eukaryotic gene expression (Sharma et at., 2019). According to the estimates, miRs regulate the expression of more than 30\% protein coding genes in eukaryotes (O'Brien et al., 2018). The crucial cellular processes like cell division, differentiation, apoptosis etc. have been shown to be controlled by miRs (Shirjang et al., 2019). Moreover, miRs are linked with the process of human tumorigenesis (Rupaimoole \& Slack, 2017). Human cancers are usually associated with altered expression patterns of one or more miRs (Wong et al., 2018). The miRs are thus valued for their prognostic role (Qadir \& Faheem, 2017). The researchers have even proposed the concept of miR-correctional theory as a vital anti-cancer strategy (Chen et al., 2018). The miR-151 has been elucidated to exhibit transcriptional downregulation or upregulation in several human cancers (Liu et al., 2019; Lv et al., 2016). The miR-151 was shown to target various protein coding genes in human cancers to modulate the growth and proliferation of cancer cells (Liu et al., 2019; Tommasi et al., 2016). Besides, its involvement in regulating cancer metastasis has been established from multiple studies (Krell et al., 2012). The miR-151 has also been shown to affect the epithelial-to-mesenchymal transition (EMT) of cancer cells (Yeh et al., 2016). Previously, the exosomal transfer of miR-151 was found to enhance the chemo-sensitivity of drug-resistant glioblastoma cells to temozolomide (Zeng et al., 2018). In the present study, miR-151 was significantly downregulated in human glioma. Low expression of miR-151 was shown to be linked with poor patient survival. The re-expression of miR-151 in glioma cells declined the growth, migration, and invasion of cancer cells. EMT was also shown to be inhibited under miR-151 overexpression. Additionally, the glioma cancer cells over-expressing miR-151 exhibited higher susceptibility to adriamycin (ADR) treatment in vitro. Profilin 2 (PFN2) was shown to act as the functional target of 
miR-151 in glioma and the regulatory effects of miR-151 in glioma were shown to be exerted through miR-151/ PFN2 axis. Collectively, the study represents the tumorsuppressive role of miR-151 in glioma and highlights its potential to enhance the chemo-sensitivity of glioma cells.

\section{MATERIALS AND METHODS}

\section{Clinical specimens}

Tissue samples, 40 glioma tissues and non-cancerous brain tissues were obtained from May 2013 to June 2016 at Tianjin Medical University General Hospital after informed consent from the patients. Further, the serum specimens were collected from peripheral blood smear from the corresponding patients, which were recognized either as high miR-151 expression group or low miR-151 group based on mean miR-151 expression. The characteristics of the patients are listed in Table 1. The study was approved by the ethics committee of Tianjin Medical University General Hospital.

\section{Cells lines and culture conditions}

The human glioma cancer cell lines (U87, U118 and $\mathrm{M} 059 \mathrm{~K}$ ) and the normal astrocytes were obtained from China Center for Type Collection (CCTCC, Wuhan, China). The cell lines were cultured at $37^{\circ} \mathrm{C}$ with $5 \% \mathrm{CO}_{2}$ in Dulbecco's modified Eagle medium (DMEM, PAN Biotech). The culture media were supplemented with $10 \%$ fetal bovine serum together with $1 \%$ penicillin/ streptomycin (PAN Biotech). For cell transfection, Lipofectamine 2000 (Thermo Fisher Scientific) was used.

\section{Expression study}

The human tissue and serum samples and cell lines were treated with TRIzol reagents to isolate the total RNA. RNA was reverse transcribed using ABI Reverse

Table 1. Clinical characteristics of glioma patients that participated in the present study

\begin{tabular}{lc}
\hline Variables & Glioma patients $(\mathrm{n}=40)$ \\
\hline Age & 17 \\
\hline$<40$ & 23 \\
\hline$>40$ & 21 \\
\hline Sex & 19 \\
\hline Male & \\
\hline Female & 4 \\
\hline WHO grades & 9 \\
\hline Grade I & 12 \\
\hline Grade II & 15 \\
\hline Grade III & \\
\hline Grade IV & 9 \\
\hline Histological sub-type & 10 \\
\hline Astrocytoma & 7 \\
\hline Anaplastic astrocytoma & 5 \\
\hline Oliodendroglioma & 8 \\
\hline Glioblastoma & 1 \\
\hline Gliocytoma & \\
\hline Meduloblastoma & \\
\hline
\end{tabular}

Table 2. List of primers used in the study.

\begin{tabular}{|c|c|c|}
\hline Primer & Direction & Sequence \\
\hline \multirow[t]{2}{*}{ miR-151 } & Forward & 5'-GGATGCTAGACTGAAGCTCCT-3' \\
\hline & Reverse & 5'-CAGTGCGTGTCGTGGAGT-3' \\
\hline \multirow[t]{2}{*}{ PFN2 } & Forward & 5'-ATGATTGTAGGAAAAGACCGGGA-3' \\
\hline & Reverse & 5'-GCAGTCACCATCGACGTATAGAC-3' \\
\hline \multirow[t]{2}{*}{ U6 } & Forward & 5'-GTCCGGTITCAGCATGTIT-3' \\
\hline & Reverse & 5'-CTCGCTTCGGCAGCACA-3' \\
\hline \multirow[t]{2}{*}{ GADPH } & Forward & 5'-CAATGACCCCTTCATTGACC -3' \\
\hline & Reverse & 5'-TGGAAGATGGTGATGGGATT -3' \\
\hline
\end{tabular}

Transcription Kit (Thermo Fisher Scientific) and cDNA was synthesized. The quantitative real-time polymerase chain reaction (qRT-PCR) was performed on QuantStudio 5.0 Real-Time PCR System (Thermo Fisher Scientific) with the help of SYBR Green PCR mix (Thermo Fisher Scientific). U6 and GAPDH were used as internal controls for miR-151 and PFN2, respectively. The relative gene expression was measured through $2^{\text {-ddCt }}$ method. The sequences of the primers used in the study are listed in Table 2.

\section{3-(4,5-dimethylthiazol-2-yl)-2,5-diphenyl tetrazolium bromide (MTT) assay}

The transfected cancer cells were placed in 96-well plates and cultured for $0,12,24,48$, or $96 \mathrm{~h}$ at $37^{\circ} \mathrm{C}$. Then, MTT solution was added, and plates were again incubated for $4 \mathrm{~h}$. After the addition of dimethyl sulfoxide (DMSO) the cells were harvested, washed with PBS, and the absorbance was measured at $570 \mathrm{~nm}$ using a micro-plate spectrometer (BioRad Laboratories).

\section{Colony formation assay}

For analyzing the colony formation, the transfected glioma cells were treated with Trypsin-EDTA solution (Sigma). The cell culturing was performed in 12-well non-adherent plates for 12 days at $37^{\circ} \mathrm{C}$. Finally, the colonies were stained with $0.1 \%$ crystal violet after being ethanol fixed. The colonies were photographed and manually counted under light microscope.

\section{5-ethynyl-2'-deoxyuridine (EdU) staining assay}

The proliferative capability of transfected glioma cells was assessed with EdU assay. The labeling of transfected glioma cells was performed using EdU labeling solution from EdU labeling/detection kit (RiboBio, China), as per the manufacturer's instructions. $70 \%$ ethanol was used for fixing the cells. The fixed cells were incubated with glycine, washed with PBS, and then administered with anti-EdU solution. The cell permeation was performed using $0.5 \%$ Triton X-100 in phosphate buffered saline (PBS). The nuclei of glioma cells were stained using $0.5 \%$ DAPI (4',6-diamidino-2-phenylindole). The fluorescent microscope was used for visualizing the cells.

\section{Wound healing assay}

The migration of glioma cells was determined with the help of wound-healing method. Briefly, the cells were cultured till confluence in 6-well plates. Subsequently, the cell surface was wounded by drawing a perpendicular scratch using pipette tip. Following $24 \mathrm{~h}$ incubation 
at $37^{\circ} \mathrm{C}$, the scratch width was observed and compared with the scratch width at $0 \mathrm{~h}$. The scratch was photographed under light microscope at $0 \mathrm{~h}$ and after $24 \mathrm{~h}$.

\section{Cell invasion assays}

The matrigel (BD biosciences) coated transwell chambers (Millipore) were prepared as per the manufacturer's instructions. Post starvation, approximately $10^{5}$ cells (in $200 \mu \mathrm{L}$ of serum-free culture medium) were placed into the top chamber. The lower chamber received only $700 \mu \mathrm{L}$ culture medium with 10\% FBS. After $24 \mathrm{~h}$ incubation at $37^{\circ} \mathrm{C}, 4 \%$ paraformaldehyde was used for fixing the cells invading the lower chamber. The staining of cells was performed with $0.1 \%$ crystal violet solution (Beyotime). Photographs were taken and cells were counted under light microscope from five randomly selected fields.

\section{Target identification and dual luciferase reporter assay}

TargetScan (http://www.targetscan.org) was used to identify the potential target of miR-151. A 3'-U'TR fragment of PFN2 was synthesized carrying either wild-type (WT) or mutant (MUT) binding site and cloned into the psiCHECK-2 reporter vector (Applied Biosystems, USA). The reporter plasmid (WT or MUT) was co-transfected with miR-151 mimics or miR-NC into U118 glioma cells. Cells were collected after $48 \mathrm{~h}$ of transfection. The Dual Luciferase Reporter Assay System (Promega, Madison, USA) was used to determine the luciferase activity.

\section{Western blotting}

The proteins from glioma cells were isolated using RIPA lysis and extraction buffer (Thermo Fisher Scientific). Then proteins were resolved on SDS-PAGE gel and transferred to PVDF membranes. After blotting, membranes were treated with primary antibodies against specific proteins at $4^{\circ} \mathrm{C}$ overnight after blocking with $5 \%$ skimmed milk. Afterwards, the membranes were exposed to horseradish peroxidase-conjugated goat anti-rabbit secondary antibody for $2 \mathrm{~h}$ at room temperature. Finally, the proteins were visualized using BeyoECL Plus Kit (Beyotime).

\section{Statistical analysis}

The statistical data were analyzed with SPSS 20.0 software and results were given as mean \pm standard deviation (S.D.). Student's $t$-test or one-way ANOVA were used for statistical analyses. The $P<0.05$ were taken as the measure of statistically significant difference.

\section{RESULTS}

\section{Downregulation of miR-151 corresponds with low patient survival}

The qRT-PCR expression analysis revealed that miR151 was significantly $(P<0.05)$ repressed in glioma tissues (Fig. 1A). The expression analysis from paired glioma and normal tissues showed that glioma tissues had significantly $(P<0.05)$ lower miR-151 expression as compared to the normal adjacent tissue specimens (Fig. 1B). The Kaplan-Meier survival analysis showed that the patients with higher miR-151 expression exhibit higher disease survival as compared to the patients with lower miR151 expression (Fig. 1C). The glioma cell lines were also seen to possess significantly lower $(P<0.05)$ miR-151

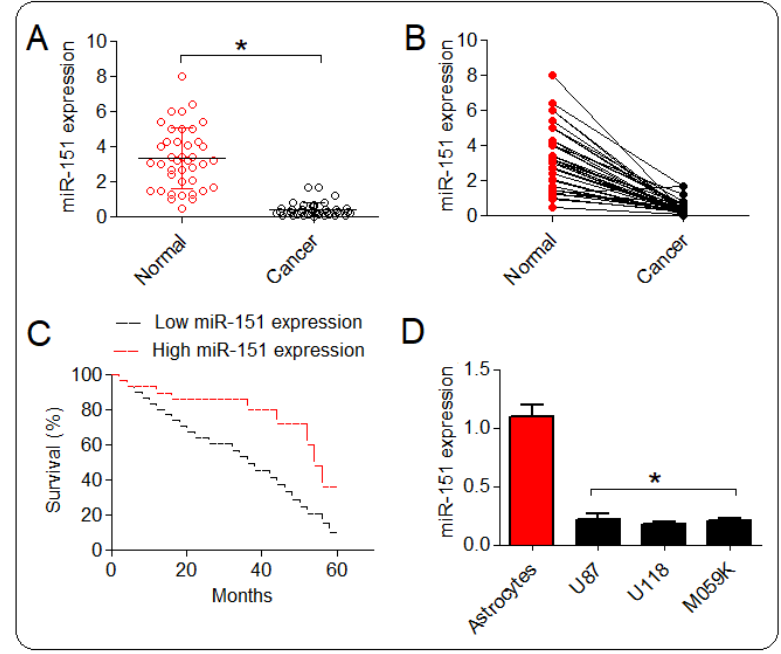

Figure 1. miR-151 is significantly repressed in glioma and predicts the patient survival.

(A) qRT-PCR analysis of miR-151 in glioma and normal adjacent tissues (B) qRT-PCR analysis of miR-151 in paired glioma and normal adjacent tissues (C) Kaplan-Meier survival analysis of glioma with reference to miR-151 expression (D) qRT-PCR expression analysis of miR-151 in glioma cell lines (U87, U118 and M059K) and normal astrocytes. The experiments represent the mean of three independent replicates \pm S.D. $\left({ }^{*} P<0.05\right)$

transcript levels (Fig. 1D) as compared to the normal astrocytes. Together, the results are suggestive of miR-151 repression in glioma, which associates with the lower patient survival.

\section{miR-151 overexpression declined the growth and viability of glioma cells}

In order to analyze the molecular role of miR-151 in glioma, miR-151 was, at first, over-expressed in U118 glioma cells (Fig. 2A). As revealed by MT'T assay, glio-

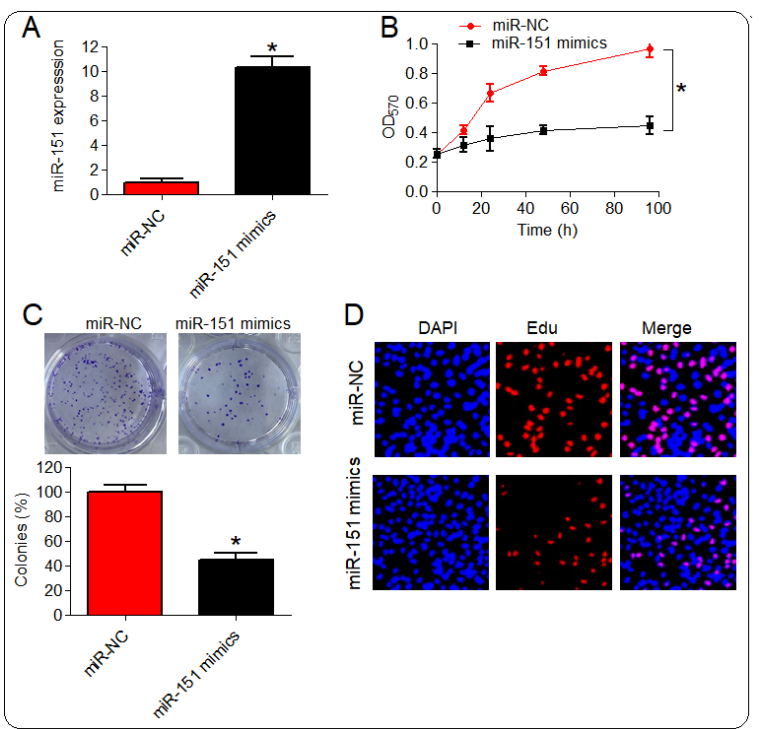

Figure 2. Glioma cell growth and viability significantly declines under miR-151 up-regulation.

(A) qRT-PCR analysis of miR-151 from U118 cells transfected with miR-151 mimics or miR-NC (B) MTT assay for the analysis of growth of U118 cells transfected with miR-151 mimics or miR-NC (C) analysis of colony formation of U118 cells transfected with miR-151 mimics or miR-NC (D) EdU assay for the analysis of proliferative viability of U118 cells transfected with miR-151 mimics or miR-NC. The experiments represent the mean of three independent replicates \pm S.D. $\left({ }^{*} P<0.05\right)$. 


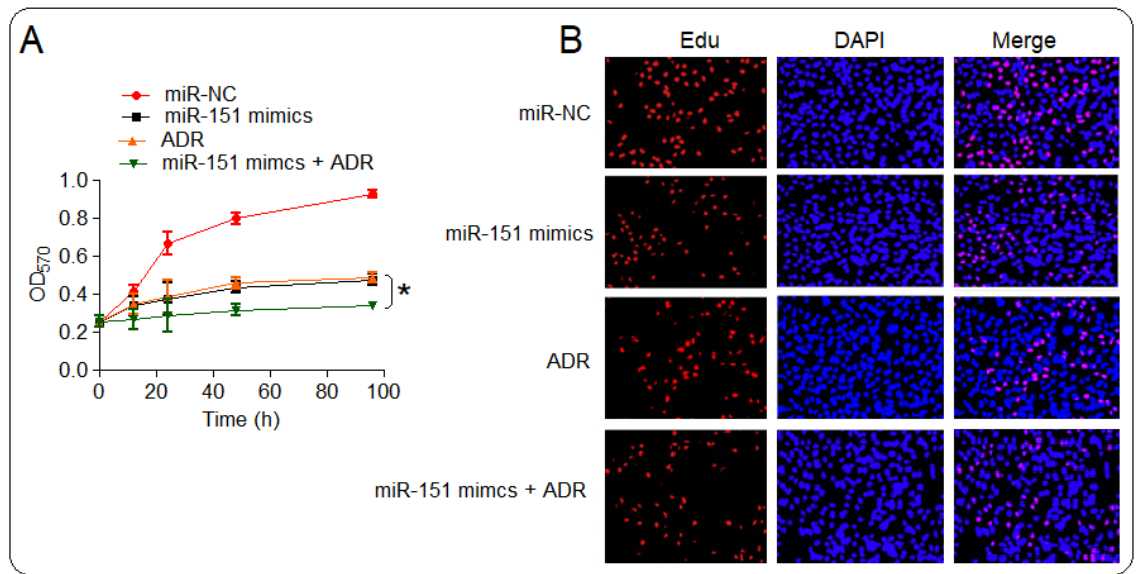

Figure 3. miR-151 overexpression enhances chemo-sensitivity of glioma cells.

(A) MTT assay for the analysis of growth of U118 cells administered with ADR, miR-151 over-expression, ADR plus miR-151 over-expression, or miR-NC control transfection (B) EdU assay showing proliferation of U118 cells administered with ADR, miR-151 over-expression, ADR plus miR-151 over-expression, or miR-NC control transfection. The experiments represent the mean of three independent replicates \pm S.D. $\left({ }^{*} P<0.05\right)$

ma cancer cells over-expressing miR-151 exhibited significantly lower growth potential in comparison to the negative control cells (Fig. 2B). The colony formation was also markedly lower under miR-151 overexpression (Fig. 2C). The EdU assay showed that glioma cells had lower proliferative ability under miR-151 overexpression (Fig. 2D). The results therefore indicate the tumor-suppressive role of miR-151 in human glioma.

\section{Glioma cells exhibited higher ADR-susceptibility under miR-151 re-expression}

To analyze if miR-151 regulates drug sensitivity of glioma cells, the glioma cells over-expressing miR-151 were treated with low dose of ADR $(10 \mu \mathrm{M})$. The glioma cells were assessed for proliferation analysis through MT'T assay with or without ADR. It was found that the overexpression of miR-151 led to higher growth inhibition of glioma cells growth by ADR than ADR only (Fig. 3A). The results were also confirmed by the EdU staining. The proportion of EdU stained cells was significantly higher when cells were treated with ADR with endogenous miR-151 expression. However, the glioma cells treated with $10 \mu \mathrm{M}$ ADR and under overexpression of miR-151 exhibited significantly lower proportion of EdU positive cells (Fig. 3B). The results therefore confirm that miR-151 overexpression sensitizes the glioma cells to external drug application.

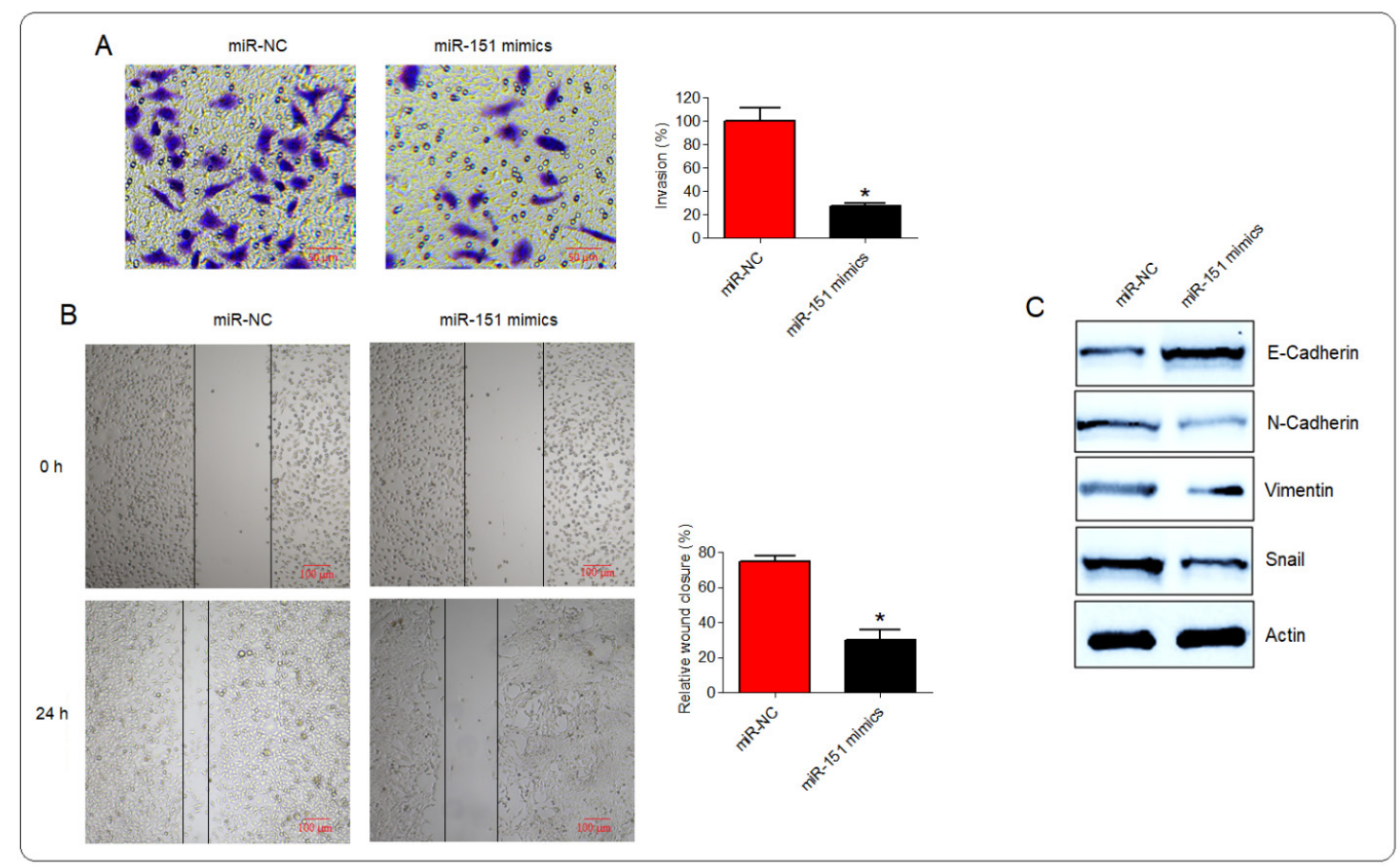

Figure 4. Invasion, migration and EMT of glioma cells is inhibited by miR-151 over-expression.

(A) Transwell assay for the analysis of invasion of glioma cells transfected with miR-151 mimics or miR-NC (B) wound-healing assay for the analysis of migration of glioma cells transfected with miR-151 mimics or miR-NC (C) western blotting of markers of EMT from glioma cells transfected with miR-151 mimics or miR-NC. The experiments represent the mean of three independent replicates \pm S.D. $\left({ }^{*} P<0.05\right)$ 


\begin{tabular}{|c|c|c|c|c|}
\hline$A$ & $\begin{array}{l}\text { Predicted consequential pairing of target region (top) } \\
\text { and miRNA (bottom) }\end{array}$ & $\begin{array}{l}\text { Site } \\
\text { type }\end{array}$ & $\begin{array}{l}\text { Context++ } \\
\text { score }\end{array}$ & $\begin{array}{l}\text { Context++ score } \\
\text { percentile }\end{array}$ \\
\hline $\begin{array}{l}\text { Position } 1489-1496 \text { of PFN2 3' UTR } 5 \\
\text { hsa-miR-151a-3p }\end{array}$ & 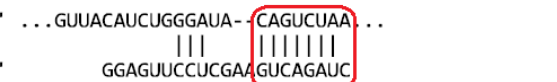 & $8 \mathrm{mer}$ & -0.50 & 99 \\
\hline
\end{tabular}

\section{B}

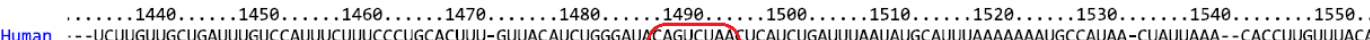
Human --.UCUUGUUGCUGAUUUGUCCAUUUCUUUCCCUGCACUUU-GUUACAUCUGGGUA CAGUCUAAA UCAUCUGAUUUAAUAUGCAUUUAAAAAAAUGCCAUAA-CUAUUAAA- - CACCUUGUUUACA Chimp ---UCUUGUUGCUGAUUUGUCCAUUUCUUUCCCUGCACUUU-GUUACAUCUGGGAUA CAGUCUAA UUCAUCUGAUUUAAUAUGCAUUUAAAAAAAUGCCAUAA-CUAUUAAA-- CACCUUGUUUACA Rhesus -- UCUUGUGCUGAUU GUCCAUUUCUUUCCCUGCACUUU-GUUACAUCUGGGAU. CAGUCUAA. UCAUCUGAUUUAUUUGCAUUUAAAAAAAUGCCAUAA-CUAUUAAA-- CACCUUGUUUACA Squirre1 --UCUUGUUGCUGAUUUGUCCAUUUCUUUCCCUGCACUUU-GUUACAUCUGGGAUA CAGUCUAA. UCAUCUGAUUUAAUAUGCAUUUAAAAAAAUGCCAUAA-CUAUUAAA--CACCUUGUUUACA Mouse -.-UCUUGUUGCUGAUUUGUCCAUUUCUUUCCCUGCACUUU-GUUACAUCUGGGAUA CAGUCUAA.UCAUCUGACUUAAUAUGCAUUUAAAAAAAUGCCAUAA-CUAUUAAA---CACCUUGUUUACA Rat ---UCUUGUUGCUGAUUUGUCCAUUUCUUUCCCUGCACUUU-GUUACAUCUGGGAUA CAGUCUAACUCAUCUGAUUUAAUAUGCAUUUAAAAAAAUGCCAUAA-CUAUUAAA--CACCUUGUUUACA Rabbit -.-UCUUGUUGCUGAUUUGUCCAUUUCUUUCCCUGCACUUU-GUUACAUCUGGGAUA CAGUCUAA UCAUCUGAUUUAAUAUGCAUUUAAAAAAAUGCCAUAA-CUAUUAAAA-CACCUUGUUUACA Pig --UCUUGUUGCUGAUUUGUCCAUUUCUUUCCCUGCACUUU-GUUACAUCUGGGAUA CAGUCUAA UUCAUCUGAUUUAAUAUGCAUUUAAAAAAAUGCCAUAA-CUAUUAAA--CACCUUGUUUACA CoW ---UCUUGUUGCUGAUUGUCCAUUUCUUUCCCUGCACUUU-GUUACAUCUGGGAUA CAGUCUAA JUCAUCUGAUUUAAUAUGCAUUUAAAAAAAUGCCAUAA-CUAUUAAA--CACCUUGUUUACA Cat ---UCUUGUUGCUGAUUGGCCAUUUCUUUCCCUGCACUUU-GUUACAUCUGGGAU CAGUCUAA JCAUCUGAUUUAAUAUGCAUUUAAAAAAAUGCCAUAA-CUAUUAAA--CACCUUGUUUACA Dog ---UCUUGUUGCUGAUUUGUCCAUUUCUUUCCCUGCACUUU-GUUACAUCUGGGAUA CAGUCUAACUCAUCUGAUUUAAUAUGCAUUUAAAAAAAUGCCAUAA-CUAUUAAA- - CACCUUGUUUACA Brown bat ---UCUUGUUGCUGAUUUGUCCAUUUCUUUCCCUGCACUUU-GUUACAUCUGGGAUACAGUCUAACUCAUCUGAUUUAAUAUGCAUUUUAAAAAAUGCCAUAA-CUAUUAAA--CACCUUGUUUACA Elephant ---UCUCGU-GCUGAUU-GUCCAUUUCUUUCCCUGCACUUU-GUUACAUCUGGGAUA CAGUCUAAFUCAUCUGAUU-AAUAUGCAUUUAAAAAAAUGCCAUAA-CUAUUAAA-- CACCUUGUUUACA

Opossum ---UCUUGUUGCUGAUUUAUCCAUUUCUUUCCCUGCACUUU-GUUACAUCUGGGAUACAGUCUAAEUCAUCUGAUUUAAUAUGCAUUUCAAAAAAUGCCAUAA-CUAUUAAAA-CACCUUGUUUACA

Macaw ---UCUUGUUGCUGAUUUGUCCAUUUCUUUCCCUGCACUUU-GUUACAUCUGGGAUA CAGUCUAAEUCAUCUGAUUUAAUAUGCAUUUCAAAAAAUGCCAUAA-CUAUUAAAAACACCUUGUUUACA

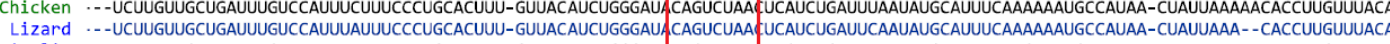
$x$. tropicalis -.--UC---UCGUUCAUUUGUCCAUU-CUUUCCCCUGCACUUUUGUUACAUCUGGGAUACAGUCUAAEUCGUCUGAUUUAAUAUGCAUUUAAAGAAAUGCCAUAAACUAUUAAAA-CACCUUGUUUACA

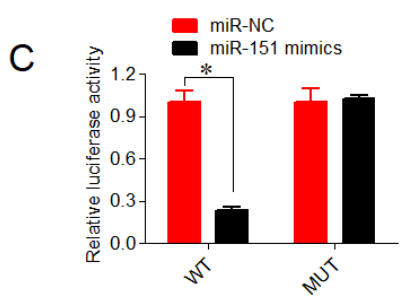

Figure 5. PFN2 was predicted as miR-151 target in glioma.

(A) Prediction of molecular target along with binding site for miR-151 (B) sequence analysis of miR-151 binding site form different animals (C) Dual luciferase assay showing the interaction between miR-151 and PFN2. The experiments represent the mean of three independent replicates \pm S.D. $\left({ }^{*} P<0.05\right)$

\section{Overexpression of miR-151 inhibited migration, invasion, and the epithelial to mesenchymal transition of human glioma cells}

From the transwell invasion assay, it was deduced that miR-151 overexpression in U118 glioma cells restricted their invasion significantly $(P<0.05)$ in comparison to the control transfection (Fig. 4A). Again, the glioma cells over-expressing miR-151 showed limited migration in comparison to the negative control cells (Fig. 4B). The analysis of expression of markers of EMT showed that miR-151 led to an increase in E-cadherin protein, while the proteins N-cadherin, Snail and Vimentin were found to decrease considerably under miR-151 overexpression (Fig. 4C). In sum, the results indicate that miR-151 negatively regulates EMT of glioma cells and thus their metastasis.

\section{PFN2 is the functional target of miR-151 in glioma}

The online bio-informatics analysis showed that miR151 interacts with 3'-UTR of profilin 2 (PFN2) at sequence site complementary with its 8 -mer nucleotide region (Fig. 5A). The sequence analysis revealed that the binding site of PFN2 3'-UTR is highly conserved in the animal kingdom (Fig. 5B). The interaction between miR151 and PFN2 was confirmed by the dual luciferase assay (Fig. 5C). The relative expression of PFN2 showed that glioma tissues exhibit significantly higher PFN2 transcript level corresponding to lower miR-151 expression levels (Fig. 6A and 6B). Further, all the glioma cell lines were shown to possess significantly higher PFN2 expression in comparison to normal astrocytes (Fig. 6C). Also, the PFN2 protein expression was significantly lower in glioma cells over-expressing miR-151 in comparison to the negative control cells (Fig. 6D). To confirm whether miR-151 regulates glioma cell growth via PFN2,
PFN2 was silenced in U118 glioma cells (Fig. 6E). The silencing of PFN2 in glioma cells remarkably reduced the cell growth like miR-151 overexpression (Fig. 6F). The overexpression of miR-151 together with PFN2 silencing led to the cell growth inhibition mimicking PFN2 silencing itself (Fig. 6G). Lastly, the upregulation of PFN2 restored the normal proliferation potential of glioma cells even under miR-151 overexpression (Fig. $6 \mathrm{H})$. The results indicate PFN2 as a function target of miR-151, which exerts the regulatory effects of the latter in glioma.

\section{DISCUSSION}

The miRs, with their tremendous potential to serve as the prognostic biomolecules in human cancer, have been proposed to act as the leading therapeutic targets against several cancer types (To et al., 2018; Qadir \& Faheem, 2017; Chen et al., 2018). There are also reports that miRs might enhance the clinical outcomes of presently employed chemo-therapy approaches (Garofalo et al., 2014). Various miRs have been shown to improve the chemo-sensitivity of cancer cells (Zeng et al., 2018; Zhang et al., 2016). In the present study, the regulatory control of miR-151 was assessed in glioma. It has been previously reported that miR-151 is repressed in glioma (Xiao et al., 2014). The results of the present study also revealed the similar inference regarding the expression pattern of miR-151 in glioma. MiR-151 was shown to act as a tumor-suppressor in human tumors such as breast cancer (Lv et al., 2016; Liu et al., 2019). The overexpression of miR-151 was reported to decline the growth and viability of cancer cells. The growth and viability of glioma cells was also affected in the similar fashion in the present study. MiR-151 was previously 


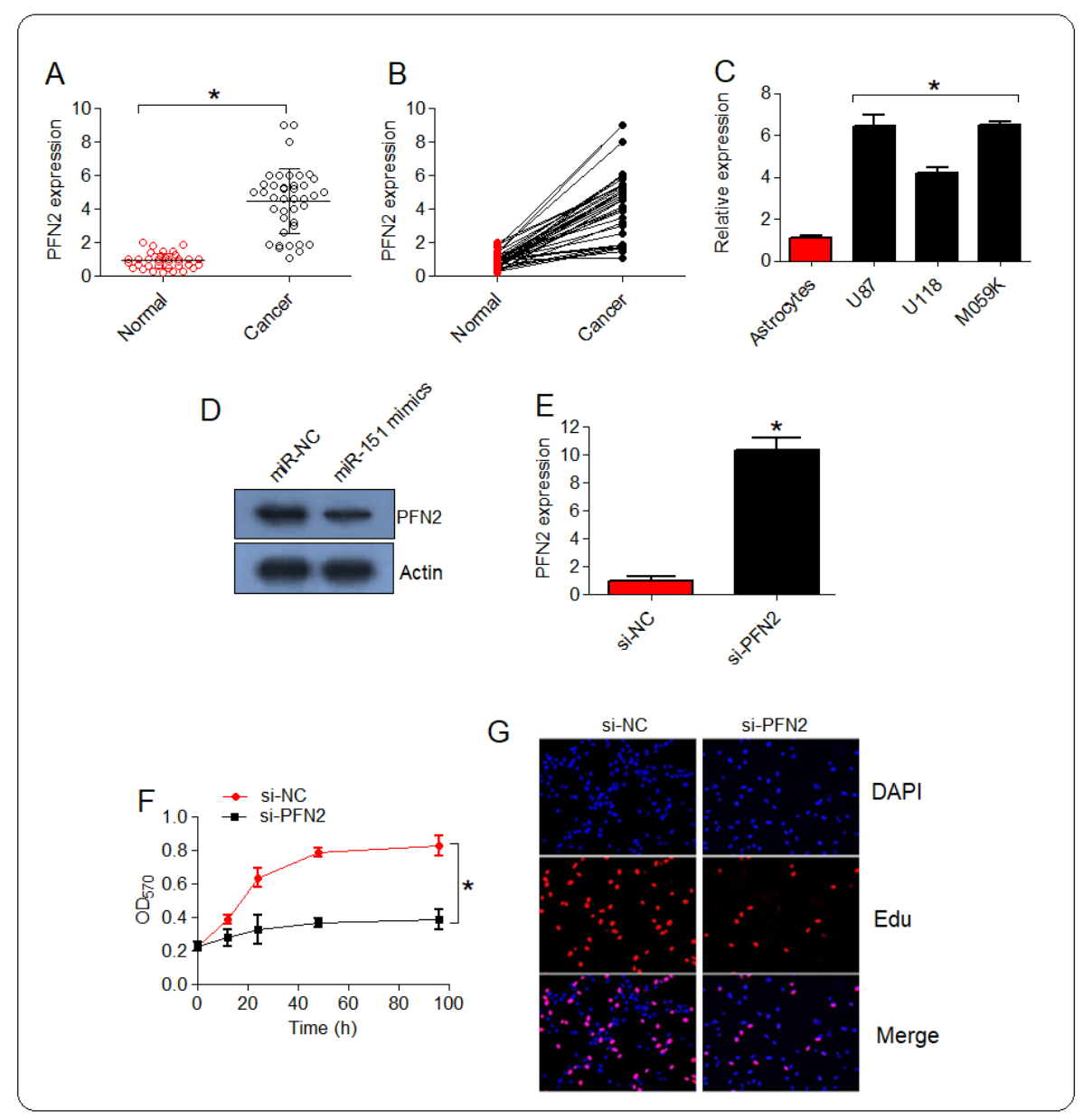

Figure 6. miR-151 targets PFN2 in glioma to exert its role.

(A) qRT-PCR analysis of PFN2 in glioma and normal adjacent tissues (B) qRT-PCR analysis of PFN2 in paired glioma and normal adjacent tissues (C) qRT-PCR expression analysis of PFN2 in glioma cell lines (U87, U118 and M059K) and normal astrocytes (D) western blotting of PFN2 from U118 glioma cells transfected with miR-151 mimics or miR-NC (E) qRT-PCR expression analysis of PFN2 from glioma cells transfected with si-PFN2 or si-NC (F) MTT assay for the analysis of growth of glioma cells transfected with si-PFN2 or si-NC (G) MTT assay for the analysis of growth of glioma cells transfected with si-PFN2, si-PFN2 plus miR-151 mimics or si-NC (H) MTT assay for the analysis of growth of glioma cells transfected with miR-151 mimics, miR-151 mimics plus pcDNA-PFN2 or miR-NC. The experiments represent the mean of three independent replicates \pm S.D. $\left({ }^{*} P<0.05\right)$

shown to increase the susceptibility of glioblastoma cells to temozolomide (TMZ) (Zeng et al., 2018). Coinciding with the same, the overexpression of miR-151 enhanced the chemo-sensitivity of glioma cells to ADR in the present study. The epithelial-to-mesenchymal transition (EMT), a complex process of cellular development, enhances the motility of cancer cells and thus enables them to invade the secondary tissues referring to metastasis (Smith \& Bhowmick, 2016). The EMT involves reduction in expression of E-cadherin protein, while $\mathrm{N}$ cadherin protein expression is seen to increase ( $\mathrm{Zhu}$ et al., 2018). The transcriptional repressor snail is reported to repress the expression of E-cadherin (Peinado et al., 2004). Vimentin, a type-III intermediate filamentary protein, is highly expressed in mesenchymal state and thus used as an EMT marker (Kokkinos et al., 2007). The miR-151 was shown to positively regulate EMT of nonsmall lung cancer cells (Daugaard et al., 2017). However, in the present study, EMT was found to be negatively regulated by miR-151, which indicates that miRs like miR-151 exhibit specific molecular roles for tissues. Evidently, the migration and invasion of glioma cells was significantly restricted by miR-151 overexpression. Similar, anti-metastatic role of miR-151 has been confirmed in the previous studies (Yeh et al., 2016). Importantly, miR-151 was shown to repress the expression of profilin 2 (PFN2) in glioma, and PFN2 was proved to act as the mediator of regulatory role of miR-151 in glioma. PFN2, a regulator of cytoskeleton, behaves like an oncogene in human tumors such as breast cancer, regulates EMT of cancer cells, and is involved in tumorigenesis of human cancers (Jaing et al., 2019; Zhou et al., 2019; Yan et al., 2017). Concluding, the miR-151 targets PFN2 in glioma and regulates the growth, metastasis, and drug sensitivity of the glioma cells. The study thus advocates the combinatorial application of miR-151 overexpression and chemo-therapy administration against glioma for better clinical outcomes.

\section{CONCLUSIONS}

The present study showed that miR-151 repression is associated with the development and progression of human glioma, suggesting that miR-151 might be utilized for the diagnosis of glioblastoma. The results further indicated the tumor-suppressive role of miR-151 against glioma together with potential to enhance the drug sensitivity of glioma cells, suggesting its therapeutic utility. 


\section{Acknowledgements}

Not applicable

\section{Conflict of interest}

All the authors declare that there is no conflict of interest.

\section{Ethical approval}

Informed consent was sought from the patients before inclusion in the present study. The research ethics committee of Tianjin Medical University General Hospital, Tianjin, China. approved the study with approval number TMU-55-2019.

\section{Availability of data and materials}

All data generated or analyzed during this study are included in this published article.

\section{Author contributions}

Conceptualization: WZ and $\mathrm{HN}$; methodology: WZ, $\mathrm{HN}$, and $\mathrm{YW}$; formal analysis and investigation: WZ, $\mathrm{HN}, \mathrm{RW}$ and CL; writing - original draft preparation: WZ and HN; writing - review and editing critically for important intellectual content: WZ; supervision: YW.

\section{REFERENCES}

Chen TH, Lee C, Chiu CT, Chu YY, Cheng HT, Hsu JT, Tsou YK, Wu RC, Chen TC, Chang NC, Yeh TS (2018) Circulating microRNA-196a is an early gastric cancer biomarker. Oncotarget 9: 1031710323. https://doi.org/10.18632/oncotarget.23126

Daugaard I, Sanders KJ, Idica A, Vittayarukskul K, Hamdorf M, Krog JD, Chow R, Jury D, Hansen LL, Hager H, Lamy P (2017) miR151a induces partial EMT by regulating E-cadherin in NSCLC cells. Oncogenesis 6: e366-e366. https://doi.org/10.1038/oncsis.2017.66

Garofalo M, Di Leva G, M Croce C (2014) MicroRNAs as anti-cancer therapy. Curr Pharm Des 20: 5328-5335. https://doi.org/10.2174/13 81612820666140128211346

Goodenberger ML, Jenkins RB (2006) Genetics of adult glioma. Cancer Genet 205: 613-621. https://doi.org/10.1016/j.cancergen.2012.10.009

Jiang M, Qiu N, Xia H, Liang H, Li H, Ao X (2019) Long non-coding RNA FOXD2-AS1/miR-150-5p/PFN2 axis regulates breast cancer malignancy and tumorigenesis. Int J Oncol 54: 1043-1052. https:// doi.org/10.3892/ijo.2019.4671

Kokkinos MI, Wafai R, Wong MK, Newgreen DF, Thompson EW, Waltham M (2007) Vimentin and epithelial-mesenchymal transition in human breast cancer - observations in vitro and in vivo. Cells Tissues Organs 185: 191-203. https://doi.org/10.1159/000101320. PMID: 17587825

Krell J, Frampton AE, Jacob J, Pellegrino L, Roca-Alonso L, Zeloof D, Alifrangis C, Lewis JS, Jiao LR, Stebbing J, Castellano L (2012) The clinico-pathologic role of microRNAs miR-9 and miR-151-5p in breast cancer metastasis. Mol Diagn Ther 16: 167-172. https://doi. org $/ 10.1007 / \mathrm{BF} 03262205$

Liu C, Li W, Zhang L, Song C, Yu H (2019) Tumor-suppressor microRNA-151-5p regulates the growth, migration and invasion of human breast cancer cells by inhibiting SCOS5. Am J Transl Res 11: 7376-7384. eCollection 2019

Lv Y, Li FL, Liu PS (2016) miR-151 promotes ovarian cancer through activation of $\mathrm{AKT} / \mathrm{mTOR}$ signaling pathway by decreasing rhogdia. Int J Clin Exp Pathol 9: 11222-11229. https://doi.org/10.3389/ fendo.2018.00402
O’Brien J, Hayder H, Zayed Y, Peng C (2018) Overview of microRNA biogenesis, mechanisms of actions, and circulation. Front Endocrinol (Lausanne) 9: 402. https://doi.org/10.3389/fendo.2018.00402

Peinado H, Ballestar E, Esteller M, Cano A (2004) Snail mediates Ecadherin repression by the recruitment of the $\operatorname{Sin} 3 \mathrm{~A} /$ histone deacetylase 1 (HDAC1)/HDAC2 complex. Mol Cell Biol 24: 306-319. https://doi.org/10.1128/MCB.24.1.306-319.2004

Qadir MI, Faheem A (2017) miRNA: A diagnostic and therapeutic tool for pancreatic cancer. Crit Rev Eukaryot Gene Expr 27: 197-204. https://doi.org/10.1615/CritRevEukaryotGeneExpr.2017019494

Rupaimoole R, Slack FJ (2017) MicroRNA therapeutics: towards a new era for the management of cancer and other diseases. Nat Rev Drug Discov 16: 203-222. https://doi.org/10.1038/nrd.2016.246

Schwartzbaum JA, Fisher JL, Aldape KD, Wrensch M (2006) Epidemiology and molecular pathology of glioma. Nat Clin Pract Neurol 2: 494-503. https://doi.org/10.1038/ncpneuro028

Sharma P, Dogra N, Singh S (2019) Small regulatory molecules acting big in cancer: potential role of mito-miRs in cancer. Curr Mol Med 19: 621-631. https://doi.org/10.2174/1566524019666190723165357

Shi C, Lamba N, Zheng LJ, Cote D, Regestein QR, Liu CM, Tran Q, Routh S, Smith TR, Mekary RA, Broekman ML (2018) Depression and survival of glioma patients: a systematic review and metaanalysis. Clin Neurol Neurosurg 172: 8-19. https://doi.org/10.1016/j. clineuro.2018.06.016

Shirjang S, Mansoori B, Asghari S, Duijf PH, Mohammadi A, Gjerstorff M, Baradaran B (2019) MicroRNAs in cancer cell death pathways: apoptosis and necroptosis. Free Radic Biol Med 139: 1-15. https://doi.org/10.1016/j.freeradbiomed.2019.05.017

Smith BN, Bhowmick NA (2016) Role of EMT in metastasis and therapy resistance. J Clin Med 5: 17. https://doi.org/10.3390/ jcm5020017

To KK, Tong CW, Wu M, Cho WC (2018) MicroRNAs in the prognosis and therapy of colorectal cancer: From bench to bedside. World J Gastroenterol 24: 2949. https://doi.org/10.3748/wjg.v24. i27.2949

Tommasi S, Pinto R, Danza K, Pilato B, Palumbo O, Micale L, De Summa S (2016) miR-151-5p, targeting chromatin remodeler SMARCA5, as a marker for the BRCAness phenotype. Oncotarget 7: 80363-80372. https://doi.org/10.18632/oncotarget.10345

Wong NW, Chen Y, Chen S, Wang X (2018) OncomiR: an online resource for exploring pan-cancer microRNA dysregulation. Bioinformatics 34: 713-715. https://doi.org/10.1093/bioinformatics/btx627

Xiao S, Yang Z, Lv R, Zhao J, Wu M, Liao Y, Liu Q (2014) miR$135 \mathrm{~b}$ contributes to the radioresistance by targeting GSK3 $\beta$ in human glioblastoma multiforme cells. PLoS One 9: e108810. https:// doi.org/10.1371/journal.pone.0108810

Yan J, Ma C, Gao Y (2017) MicroRNA-30a-5p suppresses epithelialmesenchymal transition by targeting profilin- 2 in high invasive nonsmall cell lung cancer cell lines. Oncol Rep 37: 3146-3154. https:// doi.org/10.3892/or.2017.556

Yeh TC, Huang TT, Yeh TS, Chen YR, Hsu KW, Yin PH, Lee HC, Tseng LM. (2016) miR-151-3p targets TWIST1 to repress migration of human breast cancer cells. PloS One 11: e0168171. https://doi. org/10.1371/journal.pone.0168171

Zeng A, Wei Z, Yan W, Yin J, Huang X, Zhou X, Li R, Shen F, Wu W, Wang X, You Y. (2018) Exosomal transfer of miR-151a enhances chemosensitivity to temozolomide in drug-resistant glioblastoma. Cancer Lett 436: 10-21. https://doi.org/10.1016/j.canlet.2018.08.004

Zhang Y, Hu X, Miao X, Zhu K, Cui S, Meng Q, Sun J, Wang T (2016) Micro RNA-425-5p regulates chemoresistance in colorectal cancer cells via regulation of Programmed Cell Death 10. J Cell Mol Med 20: 360-369. https://doi.org/10.1111/jcmm.12742

Zhou K, Chen J, Wu J, Xu Y, Wu Q, Yue J, Song Y, Li S, Zhou P, Tu W, Yang G (2019) Profilin 2 promotes proliferation and metastasis of head and neck cancer cells by regulating PI3K/AKT $/ \beta$ catenin signaling pathway. Oncol Res 27: 1079-1088. https://doi.org/ 10.3727/096504019X15579146061957

Zhu GJ, Song PP, Zhou H, Shen XH, Wang JG, Ma XF, Gu YJ, Liu DD, Feng AN, Qian XY, Gao X (2018) Role of epithelial-mesenchymal transition markers E-cadherin, $\mathrm{N}$-cadherin, $\beta$-catenin and ZEB2 in laryngeal squamous cell carcinoma. Oncol Lett 15: 3472 3481. https://doi.org/10.3892/ol.2018.7751 